\title{
On the Connectedness and Diameter of a Geometric Johnson Graph
}

\author{
C. Bautista-Santiago ${ }^{1}$ J. Cano ${ }^{2}$ R. Fabila-Monroy ${ }^{3}$ D. Flores-Peñaloza ${ }^{4}$ \\ $\begin{array}{lll}\text { H. González-Aguilar } & \text { D. Lara } \|^{5} & \text { E. Sarmiento }\end{array}$
}

${ }^{1}$ Dep. de Sistemas, Universidad Autónoma Metropolitana, Unidad Azcapotzalco, Mexico.

${ }^{2}$ Instituto de Matemáticas, Universidad Nacional Autónoma de México, Mexico.

${ }^{3}$ Dep. de Matemáticas, Centro de Investigación y de Estudios Avanzados del Instituto Politécnico Nacional, Mexico.

${ }^{4}$ Dep. de Matemáticas, Facultad de Ciencias, Universidad Nacional Autónoma de México, Mexico.

${ }^{5}$ Facultad de Ciencias, Universidad Autónoma de San Luis Potosím Mexico.

${ }^{6}$ Dep. de Matemàtica Aplicada II, Universitat Politècnica de Catalunya, Spain.

${ }^{7}$ Escuela Superior de Física y Matemáticas, Instituto Politécnico Nacional, Mexico

received 15 th Feb. 2012, revised $3^{\text {rd }}$ May 2013, accepted $26^{\text {th }}$ Aug. 2013.

Let $P$ be a set of $n$ points in general position in the plane. A subset $I$ of $P$ is called an island if there exists a convex set $C$ such that $I=P \cap C$. In this paper we define the generalized island Johnson graph of $P$ as the graph whose vertex consists of all islands of $P$ of cardinality $k$, two of which are adjacent if their intersection consists of exactly $l$ elements. We show that for large enough values of $n$, this graph is connected, and give upper and lower bounds on its diameter.

Keywords: Johnson graph, intersection graph, diameter, connectedness, islands.

\section{Introduction}

Let $[n]:=\{1,2, \ldots, n\}$ and let $k \leq n$ be a positive integer. A $k$-subset of a set is a subset of $k$ elements. The Johnson graph $J(n, k)$ is the graph whose vertex set consists of all $k$-subsets of $[n]$, two of which are adjacent if their intersection has size $k-1$. The Kneser graph $K(n, k)$ is the graph whose vertex set consists of all $k$-subsets of $[n]$, two of which are adjacent if they are disjoint. The generalized Johnson graph $G J(n, k, l)$ is the graph whose vertex set consists of all $k$-subsets of $[n]$, two of which are adjacent if they have exactly $l$ elements in common. Thus $G J(n, k, k-1)=J(n, k)$ and $G J(n, k, 0)=K(n, k)$.

Johnson graphs have been widely studied in the literature. This is in part for their applications in Network Design-where connectivity and diameter (i) are of importance. (Johnson graphs have small

\footnotetext{
${ }^{\dagger}$ Email: maria.dolores.lara@upc.edu

(i) A graph is connected if there is a path between any pair of its vertices. The distance between two vertices is the length of the shortest path joining them. The diameter is the maximum distance between every pair of vertices of a graph. 
diameter and high connectivity.) Geometric versions of these graphs have been defined in the literature. In Araujo et al. (2005) the chromatic numbers of some "geometric type Kneser graphs" were studied. In this paper we study the connectedness and diameter of a "geometric" version of the generalized Johnson graph.

Let $P$ be a set of $n$ points in the plane. A subset $I \subset P$ is called an island if there exists a convex set $C$ such that $I=P \cap C$. We say that $I$ is a $k$-island if it has cardinality $k$ (see Figure1). Let $0 \leq l<k \leq n$ be integers. The generalized island Johnson graph $\operatorname{IJ}(P, k, l)$ is the graph whose vertex set consists of all $k$-islands of $P$, two of which are adjacent if their intersection has exactly $l$ elements. Note that $I J(P, k, l)$ is an induced subgraph of $G J(n, k, l)$. If $P$ is in convex position, then $I J(P, k, l)$ and $G J(n, k, l)$ are isomorphic-since in this case every subset of $k$ points is a $k$-island.

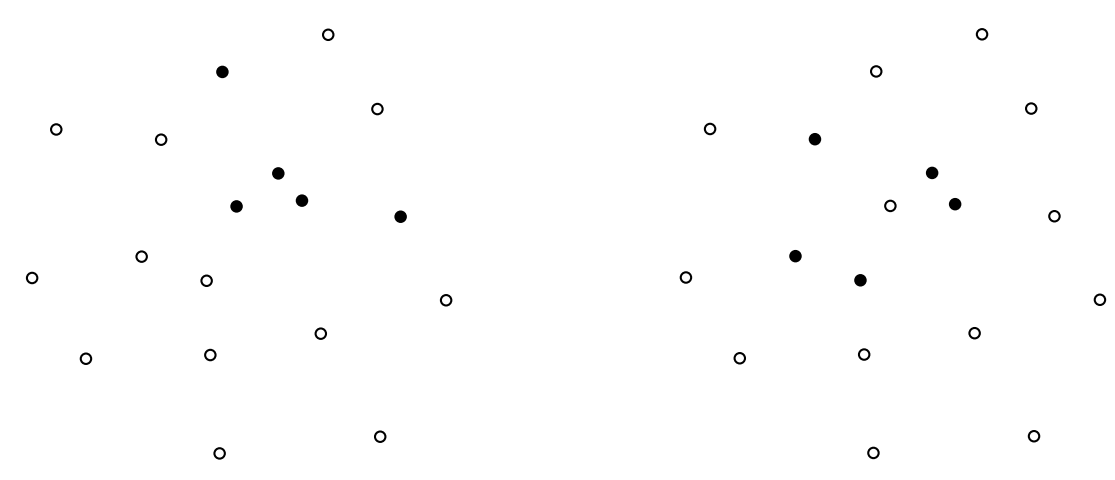

Fig. 1: A subset of 5 points which is a 5-island, and a subset of 5 points which is not (both painted black).

Graph parameters of $I J(P, k, l)$ can be translated to problems in Combinatorial Geometry of point sets. Here are some examples.

- The number of vertices of this graph is the number of $k$-islands of $P$-the problem of estimating this number was recently studied in Fabila-Monroy and Huemer (2011).

- An empty triangle of $P$ is a triangle with vertices on $P$ and without points of $P$ in its interior. The empty triangles of $P$ are precisely its 3 -islands (or the number of vertices in $I J(P, 3, l)$ ). Counting them has been a widely studied problem Bárány and Füredi (1987); Bárány and Valtr (2004); Dumitrescu (2000); Katchalski and Meir (1988); Valtr (1995).

- A related question Bárány and Károlyi (2001) is: What is the maximum number of empty triangles that can share an edge? This translates to the problem of determining the clique number of $I J(P, 3,2)$.

The paper is organized as follows. In Section 2, we prove that $I J(P, k, l)$ is connected when $n$ is large enough with respect to $k$ and $l$. The proof of this result implies an upper bound of $O\left(\frac{n}{k-l}\right)+O(k-l)$ on the diameter of this graph. In Section 3 , we improve this bound for the case when $l \leq k / 2$, where we show that the diameter is at most $O(\log n)+O(k-l)$. We also exhibit a choice of $P$ for which $I J(P, k, l)$ has diameter at least $\Omega\left(\frac{\log n-\log k}{\log (k-l)}\right)$. Note that these bounds are asymptotically tight when $l \leq k / 2$ and, 


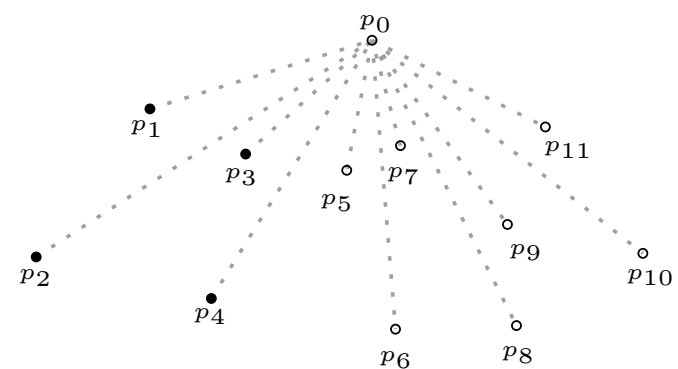

(a) Projectable.

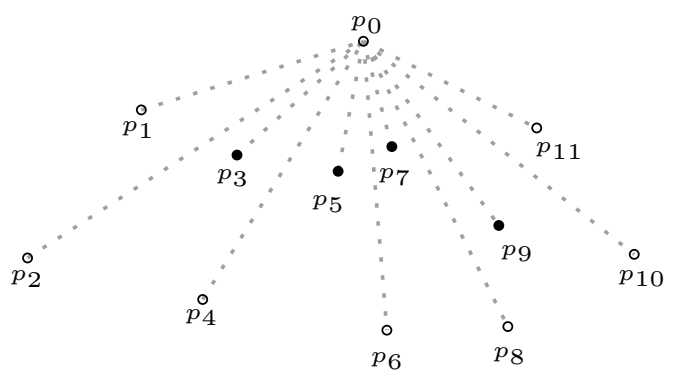

(b) Non projectable.

Fig. 2: A 4-island which is projectable, and a 4-island which is not projectable.

$k$ and $l$ are constant with respect to $n$. A preliminary version of this paper appeared in Bautista-Santiago et al. (2010).

\section{Connectedness}

In this section we prove the following theorem.

Theorem 2.1 If $n>(k-l)(k-l+1)+k$, then $I J(P, k, l)$ is connected and its diameter is $O\left(\frac{n}{k-l}\right)+$ $O(k-l)$.

The proof is divided in two parts:

- First we choose $\mathcal{F}$, an appropriate 'core' subgraph from $I J(P, k, l)$ which has small diameter and is connected.

- Next we prove that for every vertex in $I J(P, k, l)$ there is a path of length at most $O\left(\frac{n}{k-l}\right)$ connecting it to a vertex in $\mathcal{F}$.

\subsection{Choosing a core subgraph from $I J(P, k, l)$}

Let $P:=\left\{p_{0}, p_{1}, \ldots, p_{n-1}\right\}$ be a set of $n$ points in general position in the plane. So that $p_{0}$ is the topmost point of $P$, and $p_{1}, \ldots, p_{n-1}$ are sorted counterclockwise by angle around $p_{0}$. For $0 \leq i \leq j \leq n$, let $P_{i, j}:=\left\{p_{i}, p_{i+1}, \ldots, p_{j}\right\}$ and let $P_{i, j}^{\prime}:=P_{i, j} \cup\left\{p_{0}\right\}$. Observe that $P_{i, j}$ and $P_{i, j}^{\prime}$ are both islands of $P$. We call these two types of islands projectable, and define this concept next.

Definition 2.2 The projection of a point $p_{i}, i \neq 0$, is the intersection of the ray emanating from $p_{0}$ and passing through $p_{i}$ with a horizontal line. The projection of an island is the projection of its point set after omitting $p_{0}$. Projectable islands are those islands that can be "projected" in such a way that its points are consecutive in the image of the whole set; see Figure 2

Let $\mathcal{F}$ be the subgraph of $I J(P, k, l)$ induced by the projectable $k$-islands of $P$. Let $S$ be a set of $n-1$ points on a horizontal line $h$, and let $S^{\prime}:=S \cup\{x\}$, where $x$ is a point not in $h$. It is not hard to see that $\mathcal{F}$ is isomorphic to $I J\left(S^{\prime}, k, l\right)$. We classify the islands of $S^{\prime}$ into two types: those that contain $x$, and those that do not. Notice that these two types correspond to the two types of projectable islands of $P$. 
Now we show that $I J\left(S^{\prime}, k, l\right)$ is connected. First we consider the subgraph of $I J\left(S^{\prime}, k, l\right)$ induced by those islands of $S^{\prime}$ that do not contain $x$. Note that this is precisely $I J(S, k, l)$. Without loss of generality assume that $S$ is a set $x_{1}<x_{2}<\cdots<x_{n-1}$ of points on the real line. Observe that a $k$-island of $S$ is an interval of $k$ consecutive elements $\left\{x_{i}, \ldots, x_{i+k-1}\right\}$. For the sake of clarity, in what follows we refer to $k$-islands of $S$ as $k$-intervals.

Two $k$-intervals of $S$ are adjacent in $I J(S, k, l)$ if they overlap in exactly $l$ elements. It follows easily that if $l>0$, each $k$-interval is adjacent to at most two different $k$-intervals, one containing its first element, and the other containing its last; see Figure 3 . Since $I J(S, k, l)$ has no cycles and its maximum degree is at most two, it is a union of pairwise disjoint paths. These paths can be described as follows. For $i<j$, let $A_{i}$ and $A_{j}$ be the intervals ending at $x_{i}$ and $x_{j}$ respectively. There is a path between $A_{i}$ and $A_{j}$ if and only if $i \equiv j \bmod (k-l)$. Consider the interval adjacent with $A_{i}$ to its right, this interval must end at point $x_{i+(k-l)}$ (leaving exactly $l$ points on the intersection). On the other hand, the interval adjacent with $A_{i}$ to its left, must end at point $x_{i-(k-l)}$; see Figure 3 For $0 \leq r<k-l$, let $\mathcal{P}_{r}$ be the subgraph of $I J(S, k, l)$ induced by those $k$-intervals ending at a point with index congruent to $r \bmod (k-l)$. Thus we have:

Proposition 2.3 If $l>0, \mathcal{P}_{r}$ is an induced path of $I J(S, k, l)$. Moreover $I J(S, k, l)$ is the union of $\left\{\mathcal{P}_{r} \mid 0 \leq r<k-l\right\}$.

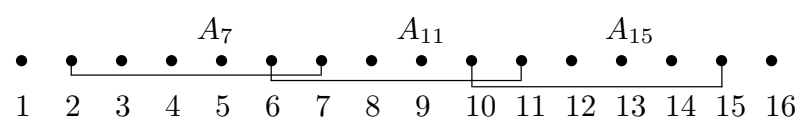

Fig. 3: Three different $k$-intervals, for $|S|=16, k=6, l=2$.

For $l=0$ and $n \geq 3 k-1$, every $k$-interval would either intersect the left-most $k$-interval or the rightmost $k$-interval, but not both. In this case $I J(S, k, 0)$ is connected and its diameter is at most 3 . Note that except for some special cases, $I J(S, k, l)$ is disconnected. Remarkably, as we show next, for a large enough value of $n$, the addition of one extra point makes the graph connected.

As before $A_{i}$ is the $k$-island ( $k$-interval) that ends at point $x_{i}$ and does not contain $x$. Let $A_{i}^{\prime}$ be the $k$-island ending at point $x_{i}$ and containing $x$; see Figure 4 . The structure of $I J\left(S^{\prime}, k, l\right)$ when $l<2$ is different from when $l \geq 2$. In what follows, we assume that $l \geq 2$ and briefly discuss the case $l<2$ at the end of this section. Note that the subgraph of $I J\left(S^{\prime}, k, l\right)$ induced by the $A_{i}$ 's is precisely $I J(S, k, l)$; and the subgraph induced by the islands $A_{i}^{\prime}$ is isomorphic to $I J(S, k-1, l-1)$. From these observations, the following lemma is not hard to prove:

Lemma 2.4 If $l \geq 2$, then in $I J\left(S^{\prime}, k, l\right)$ :

1. $A_{i}$ is adjacent to $A_{i-(k-l)}^{\prime}$ and $A_{i+(k-l)-1}^{\prime}$ (if they exist).

2. $A_{i}^{\prime}$ is adjacent to $A_{i+(k-l)}$ and $A_{i-(k-l)+1}$ (if they exist).

The following theorem provides sufficient and necessary conditions for $I J\left(S^{\prime}, k, l\right)$ to be connected.

Theorem 2.5 For $l \geq 2$, the graph $I J\left(S^{\prime}, k, l\right)$ is connected if and only if $n \geq 3 k-2 l-1$ or $n=k$. 


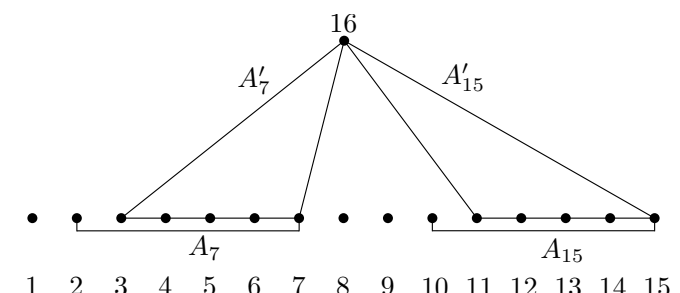

Fig. 4: Four different $k$-islands in $S^{\prime}$, for $\left|S^{\prime}\right|=16, k=6, l=2$.

Proof: Let $I$ and $J$ be two $k$-islands of $S^{\prime}$. As long as the intermediate islands exists we can repeatedly use Lemma 2.4 to find a path from I to an island whose endpoint is in the same residue class of $(k-l)$ as the endpoint of $J$, and that contains $x$ if and only if $J$ does. This is the case whenever $n \geq 3 k-2 l-1$ : Consider the set of islands $\left\{A_{k}, A_{k+1}, A_{k+2}, \ldots, A_{k+(k-l-1)}\right\}$. Notice that, for any value of $k$ and $l \geq 2$, all islands in this set exist. Furthermore, there is exactly one island in the set for each residue class. Afterwards Proposition 2.3 ensures that there is a path from this island to J.

Suppose that $n<3 k-2 l-1$, then there exists a $k$-island containing $x$ and having less than $k-l$ points to its left and less than $k-l$ points to its right. This island is an isolated vertex in $I J\left(S^{\prime}, k, l\right)$. This sole vertex is all of $I J\left(S^{\prime}, k, l\right)$ when $n=k$ (in which case the graph is connected). However, if $n>k$, there are at least two such $k$-islands.

The proof of Theorem 2.5 implicitly provides the following bound on the diameter of $I J\left(S^{\prime}, k, l\right)$.

Proposition 2.6 If I $J\left(S^{\prime}, k, l\right)$ is connected, then its diameter is $O\left(\frac{n-k}{k-l}\right)+O(k-l)$.

Proof: Suppose that $n \geq 3 k-2 l-1$, as otherwise the bound trivially holds. Let I and J be two $k$-islands of $S^{\prime}$. Note that it takes at most $2(k-l)$ applications of Lemma 2.4 to take I to an island whose endpoint is in the same residue class of $(k-l)$ as the endpoint of $J$, and that contains $x$ if and only if $J$ does. The path in $I J\left(S^{\prime}, k, l\right)$ or in $I J(S, k, l)$-depending on whether $J$ contains $x$ or not-connecting this island to $J$ has length at most $\left\lceil\frac{n-k}{k-l}\right\rceil$.

Finally we consider the case when $l<2$. As we mentioned before, if $l=0$ and $n \geq 3 k-1, I J(S, k, 0)$ is connected and its diameter is at most 3 . This is the case also for $I J\left(S^{\prime}, k, 0\right)$. On the other hand, if $l=1$, then the islands containing $x$ induce a graph isomorphic to $I J(S, k-1,0)$. From these observations and Lemma2.4, we get the following result.

Proposition 2.7 If $n \geq 3 k, I J\left(S^{\prime}, k, 0\right)$ and $I J\left(S^{\prime}, k, 1\right)$ are connected and of diameter at most 4 .

\subsection{Paths between projectable and non projectable islands}

To finish the proof of Theorem 2.1, we prove that for any island of $P$, there is a path connecting it to a projectable island. At the end of this section we present a first bound on the diameter of $I J(P, k, l)$.

Recall that $P=\left\{p_{0}, p_{1}, \ldots, p_{n-1}\right\} ; p 0$ is its topmost point and $p_{1}, \ldots, p_{n-1}$ are sorted counterclockwise by angle around $p_{0}$. Let $A$ be an island of $P$ such that $\left|A \backslash\left\{p_{0}\right\}\right| \geq 2$. Define the weight of $A$ as the difference between the largest and the smallest indices of the elements of $A \backslash\left\{p_{0}\right\}$-an island of weight 
$k-1$ is always projectable. The following lemma ensures the existence of a path between any island and a projectable island, by eventually reducing the weight of any given island.

Lemma 2.8 (Shrinking Lemma). If $n>(k-l)(k-l+1)+k$, then every non projectable $k$-island $A$ of $P$ has a neighbor in $I J(P, k, l)$ which is either a projectable island or an island whose weight is less than that of $A$ by at least $k-l$.

Proof: Let the elements of $A$ different from $p_{0}$ be $p_{i_{1}}, \ldots, p_{i_{m}}$. Thus $m$ is equal to $k$ or to $k-1$ depending on whether A contains $p_{0}$ or not. Consider all maximal intervals of $P \backslash\left\{p_{0}\right\}$ containing exactly l elements of $A$. (That is maximal sets of consecutive elements of $P \backslash p_{0}$ containing exactly l elements of $A$.)

We distinguish two of these intervals: the one containing the first point of $P \backslash\left\{p_{0}\right\}$ and the one containing the last. We refer to them as end intervals, and to the rest as interior intervals.

Note that there are at most $k-l+1$ such intervals and that every element of $P \backslash\left\{p_{0}\right\}$ is in at least one of them. Since $n>(k-l)(k-l+1)+k$, one of these intervals, $I$, must contain at least $(k-l)$ points of $P \backslash A$.

Suppose that $I$ is an interior interval. Let $J:=A \cap I$, note that $|J|=l$ (if $l=0$, set $J:=\emptyset$ ). If $J$ is non empty let $B$ be the set of the $k-l$ points of $I \backslash A$ closes ${ }^{\text {(ii) }}$ to $C o n v(J)$. If $J$ is empty then let $B$ be any $k$-island contained in $I \backslash A$. Then $J \cup B$ is a $k$-island adjacent to $A$ in $I J(P, k, l)$, and its weight is smaller than the weight of $A$ by at least $k-l$.

Now suppose that $I$ is an end interval, let $p_{S}$ and $p_{E}$ be the first and last elements in $A \cap I$. If $\left[p_{S}, p_{E}\right]$ contains at least $k-l$ elements of $P \backslash A$, then proceed as with interior intervals. Otherwise, there are $r<k-l$ points of $P \backslash A$ in I. If I is the first interval, then let $B$ be the $k-l-r$ points previous to $p_{S}$ in $P \backslash\left\{p_{0}\right\}$. If $I$ is the last interval, then let $B$ be the $k-l-r$ points after $p_{E}$. Note that in either case, $\left[p_{S}, p_{E}\right] \cup B$ is a projectable island adjacent to $A$.

We are ready to finish the proof of Theorem 2.1.

Theorem 2.1 If $n>(k-l)(k-l+1)+k$, then $I J(P, k, l)$ is connected and its diameter is $O\left(\frac{n}{k-l}\right)+$ $O(k-l)$.

Proof: Let $A$ and $B$ be $k$-islands of $P$. We apply Lemma 2.8 successively to find a sequence of consecutive adjacent islands $A=A_{0}, A_{1}, \ldots, A_{m}$ and $B=B_{0}, B_{1}, \ldots, B_{m^{\prime}}$, in which each element has weight smaller than the previous by at least $k-l$, and the last element is a projectable island. Since the weight of the initial terms is at most $n$, these sequences have length $O\left(\frac{n}{k-l}\right)$.

As noted before the subgraph induced by the projectable islands is isomorphic to IJ $\left(S^{\prime}, k, l\right)$. Simple arithmetic shows that if $n>(k-l)(k-l+1)+k$, then $n>3 k-2 l-2$. Thus this subgraph is connected and has diameter $O\left(\frac{n-k}{k-l}\right)+O(k-l)$ (Theorem 2.5 and Proposition 2.6. Hence the diameter of $I J(P, k, l)$ is $O\left(\frac{n}{k-l}\right)+O(k-l)$ as claimed.

(ii) The distance between $\operatorname{Conv}(J)$ and a point $p \notin \operatorname{Conv}(J)$, is defined as the length of the shortest line segment having $p$ and a point of $\operatorname{Conv}(J)$ as endpoints. 


\section{Bounds}

\subsection{Upper bound}

In this section, for the case when $l \leq k / 2$, we improve the upper bound on the diameter of $I J(P, k, l)$ given in Theorem 2.1 We use a divide and conquer strategy. Let $A$ and $B$ be two vertices of $I J(P, k, l)$. First we find a neighbor of $A$ and a neighbor of $B$; discarding half of the points of $P$ in the process. We iterate on the new found neighbors. Just before $P$ has very few points and $I J(P, k, l)$ may be disconnected; we apply Theorem 2.1.

The following lemma provides the divide and conquer part of the argument. The proof uses some of the ideas of the proof of the Shrinking Lemma.

Lemma 3.1 Let $A$ and $B$ be two vertices of $I J(P, k, l)$. If $n \geq 2((k-l)(k-l+1)+k)$, and $l \leq k / 2$, then there exists a closed halfplane, $H$, containing at most $n / 2$ and at least $(k-l)(k-l+1)+k$ points of $P$. With the additional property that $A$ and $B$, each have a neighbor contained entirely in $H$.

Proof: We use the ham-sandwich theorem to find a line $\ell$ so that each of the two closed halfplanes bounded $\ell$ by contain $\lceil k / 2\rceil$ points of $A$ and $\lceil k / 2\rceil$ points of $B$.

Without loss of generality suppose that the halfplane $H$ above $\ell$ contains at most $n / 2$ points of $P$. If $H$, however, does not contain at least $(k-l)(k-l+1)+k$ points of $P$, move $\ell$ parallel down until it does. In this case $H$ would contain at least as many points of $A$ and $B$ as it previously did and since we are assuming that $n \geq 2((k-l)(k-l+1)+k)$, it still contains at most $n / 2$ points of $P$.

We will now show the existence of a neighbor of $A$ in $I J(P, k, l)$ with the desired properties. The corresponding neighbor of $B$ can be found in a similar way. Let $P:=P \cap H$ and sort its elements by distance to $\ell$. As in the proof of Lemma 2.8 we consider maximal intervals of $P^{\prime}$ containing exactly $l$ consecutive elements of $A$. There is at least one such interval, given that $H$ contains at least $k / 2$ points of $A$ and that we are assuming $l \leq k / 2$. The rest of the proof employs the same arguments as the proof of Lemma 2.8 to find a neighbor of A contained in one of these intervals.

Theorem 3.2 If $n \geq 2((k-l)(k-l+1)+k)$ and $l \leq k / 2$, then the diameter of $I J(P, k, l)$ is $O(\log n)+$ $O(k-l)$.

Proof: Consider the following algorithm. Let $A$ and $B$ be two k-islands of $P$. We start by setting $A_{0}:=A, B_{0}:=B, P_{0}:=P, n_{0}:=n$. While $n_{i} \geq 2((k-l)(k-l+1)+k)$, we apply Lemma 3.1 to $P_{i}, A_{i}$, and $B_{i}$. At each step we obtain a closed halfplane $H_{i}$ containing at most $n_{i} / 2$ and at least $(k-l)(k-l+1)+k$ points of $P_{i}$, with the additional property that both $A_{i}$ and $B_{i}$ have neighbors $A_{i+1}$ and $B_{i+1}$ contained entirely in $H_{i}$. We set $P_{i+1}:=H_{i} \cap P_{i}, n_{i+1}:=\left|P_{i+1}\right|$, and continue the iteration. We can do this procedure at most $O(\log n)$ times. In the last iteration, we have a point set $P_{m}$ with fewer than $2((k-l)(k-l+1)+k)$ and at least $(k-l)(k-l+1)+k$ elements. The islands $A_{m}$ and $B_{m}$ are both contained in $P_{m}$, and are joined by paths of length $O(\log n)$ to $A$ and $B$ respectively. We apply Theorem 2.1 to obtain a path of length at most $O(k-l)$ from $A_{m}$ to $B_{m}$. Concatenating the three paths we obtain a path of length $O(\log n)+O(k-l)$ from $A$ to $B$ in $I J(P, k, l)$.

\subsection{Lower bound}

For the lower bound we use Horton sets Horton (1983). We base our exposition on Matoušek (2002). Let $X$ and $Y$ be two point sets in the plane. We say that $X$ is high above $Y$ (and that $Y$ is deep below $X$ ), if the following conditions are met: 
- No line passing through a pair of points of $X \cup Y$ is vertical.

- Each line passing through a pair of points of $X$ lies above all the points of $Y$.

- Each line passing through a pair of points of $Y$ lies below all the points of $X$.

For a set $X=\left\{x_{1}, x_{2}, \ldots, x_{n}\right\}$ of points in the plane with no two points having the same $x$-coordinate and with the indices chosen so that the $x$-coordinate of $x_{i}$ increases with $i$, we define the sets $X_{0}=$ $\left\{x_{2}, x_{4}, \ldots\right\}$ (consisting of the points with even indices) and $X_{1}=\left\{x_{1}, x_{3}, \ldots\right\}$ (consisting of the points with odd indices). Thus $X_{00}=\left\{x_{4}, x_{8}, \ldots\right\}, X_{01}=\left\{x_{2}, x_{6}, \ldots\right\}, X_{10}=\left\{x_{3}, x_{7}, \ldots\right\}$ and $X_{11}=$ $\left\{x_{1}, x_{5}, \ldots\right\}$.

Definition 3.3 A finite set of points $H_{0}$, with no two of points having the same $x$-coordinate, is said to be a Horton set if $\left|H_{0}\right| \leq 1$, or the following conditions are met:

- Both $H_{00}$ and $H_{01}$ are Horton sets.

- $H_{00}$ is high above $H_{01}$.

Horton sets of any size were shown to exist in Horton (1983). We remark that in Matoušek (2002), in the definition of Horton sets, the second condition is that " $H_{00}$ is high above $H_{01}$ or $H_{01}$ is high above $H_{00}$ ". For our purposes we need to fix one of these two options.

Let $H_{0}:=\left\{x_{1}, \ldots, x_{n}\right\}$ be a Horton set of $n$ points. Given an island $A$ of $H_{0}$, we define its depth, $\delta(A)$, to be the length of the longest string $s:=00 \ldots 0$ of all zeros such that $H_{s}$ contains $A$. Thus for example, $\delta\left(x_{1}\right)=1, \delta\left(x_{2}\right)=2, \delta\left(x_{3}\right)=1, \delta\left(x_{4}\right)=3, \cdots ;$ refer to Figure 5 Note that the depth of an island is the depth of its shallowest point.

Lemma 3.4 Let $x$ and $y$ be two points of $H_{0}$, such that $x$ is to the left of $y$, and $z$ is a point with depth less than $\delta(\{x, y\})$. Then the island $H_{0} \cap \operatorname{Conv}(\{x, y, z\})$ contains at least $2^{\delta(\{x, y\})-\delta(z)-1}-1$ points with depth greater than that of $z$, and lying in between $x$ and $y$.

Proof: Let $A:=H_{0} \cap \operatorname{Conv}(\{x, y, z\})$. We will proceed by induction on $r=\delta(\{x, y\})-\delta(z)$. If $r=1$ there is nothing to prove, since $2^{r-1}-1=0$. Assume then that $r>1$. Let $s$ be the unique string of all zeros, such that $H_{s}$ contains $A$ but $H_{s 0}$ and $H_{s 1}$ do not. Note that $z$ lies in $H_{s 1}$ while $x$ and $y$ both lie in $H_{s 0}$; actually since we are assuming $r>1$, they both lie in $H_{s 00}$. Consider the set $H_{s 0}$, since it is a Horton set, A contains at least a point in $H_{s 01}$, between $x$ and $y$. Of all such points, choose $x_{k}^{\prime}$ to be the shallowest. The depth of $x_{k}^{\prime}$ is one more than that of $z$. By induction, the island $H_{0} \cap \operatorname{Conv}\left(\left\{x, y, x_{k}^{\prime}\right\}\right)$ contains a set I of at least $2^{r-2}-1$ points. These points have depth greater than that of $x_{k}^{\prime}$ (thus contained in $H_{s 00}$ ) and lay between $x$ and $y$. Therefore $I$ is contained in A. For each point in $I$, consider the next point $x_{m}$ to its right in $H_{s} 0$. This point must be in $H_{s} 01$ and $\delta(z)<\delta\left(x_{m}\right)$. Thus we have $2^{r-2}-1$ additional points in A. Note that the point to the right of $x$ in $H_{s} 0$ is not in the previous counting. Therefore $A$ has at least $2^{r-2}-1+2^{r-2}-1+1=2^{r-1}-1$ points with depth greater than that of $z$, and lying between $x$ and $y$.

Lemma 3.5 If $A$ and $B$ are two adjacent islands in $I J\left(H_{0}, k, l\right)$ (with $\left.l \geq 2\right)$, then their depths differ by at most $O(\log (k-l))$.

Proof: Without loss of generality assume that the depth of $A$ is greater than the depth of $B$. Let $C$ be the island $A \cap B$. Note that the depth of $C$ is at least the depth of $A$. If $z$ is the shallowest point of $B$, 
On the Connectedness and Diameter of a Geometric Johnson Graph
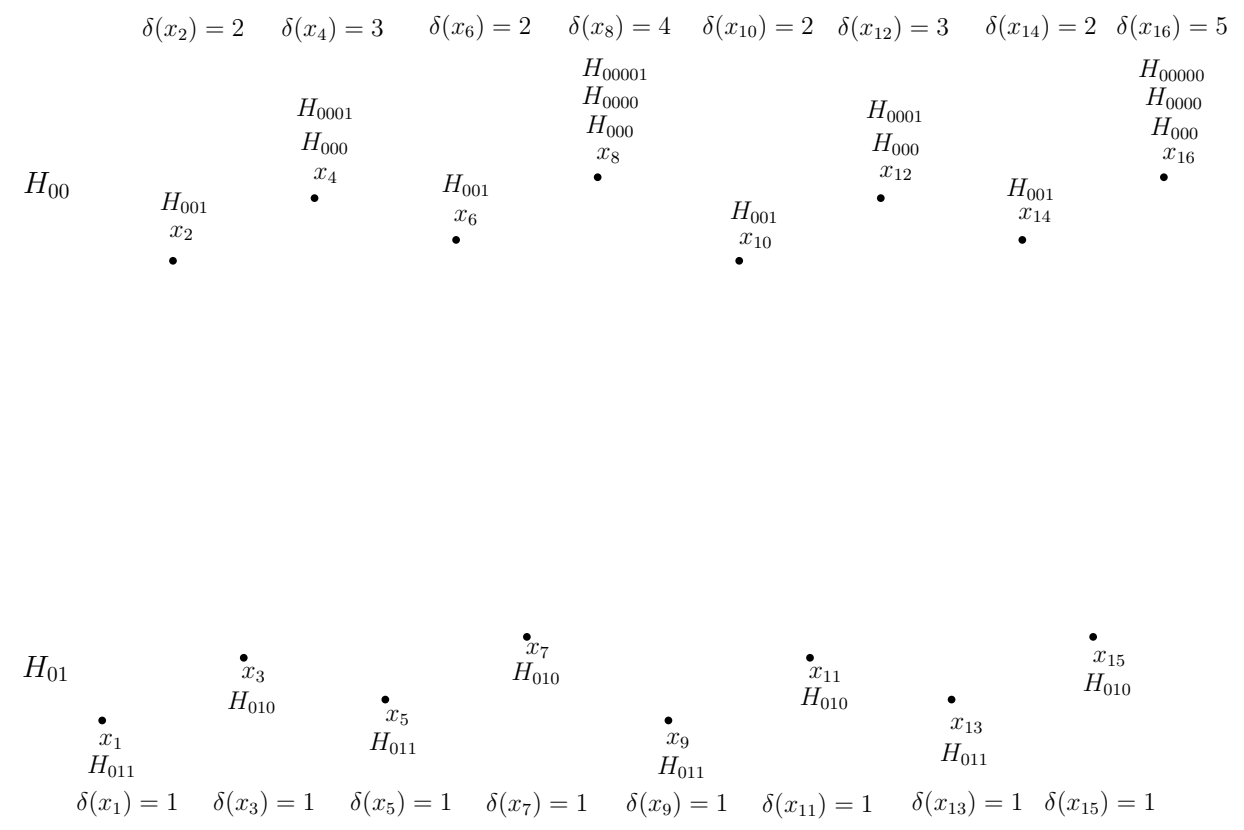

Fig. 5: A Horton set with 16 points, and the depth of its elements.

then $\delta(z)=\delta(B)$, and this point has depth less than $\delta(C)$. Consider an edge of the convex hull of $C$, whose supporting line separates $C$ and $z$. Let $x$ and $y$ be its endpoints. Then by Lemma 3.4 the island $H_{0} \cap \operatorname{Conv}(\{x, y, z\})$ contains at least $2^{\delta(\{x, y\})-\delta(z)-1}-1 \geq 2^{\delta(A)-\delta(B)-1}-1$ points, none of which is in $C$. However, since these points do lie in $B$, there are at most $k-l$ of them. Therefore $\delta(A)-\delta(B)$ is $O(\log (k-l))$ as claimed.

Theorem 3.6 The diameter of $I J\left(H_{0}, k, l\right)$ for $l \geq 2$ is $\Omega\left(\frac{\log n-\log k}{\log (k-l)}\right)$

Proof: Let $A$ be an island with the largest possible depth, which is $\left\lceil\log _{2} n / k\right\rceil$. Let $B$ be an island of depth 1. By Lemma 3.5 in any path joining $A$ and $B$ in $I J\left(H_{0}, k, l\right)$, the depth of two consecutive vertices differs by $O(\log (k-l))$. Therefore any such path must have length $\Omega\left(\frac{\log n-\log k}{\log (k-l)}\right)$.

We point out that there was an error in the proof of Theorem 11 in the preliminary version of this paper Bautista-Santiago et al. (2010); thus the bounds stated there are incorrect.

The diameter of the generalized Johnson graph can be substantially different from that of the generalized island Johnson graph. The diameter of $G J(n, k, l)$ is $O(k)$ when $n$ is large enough, while the diameter of $I J(P, k, l)$ can be $\Omega\left(\frac{\log n-\log k}{\log (k-l)}\right)$.

Determining upper and lower bounds for the diameter of $I J(P, k, l)$ seems to be a challenging problem when $l>k / 2$. It might happen that there is a sharp jump in the diameter when $l$ rises above $k / 2$. We leave the closing of this gap as an open problem. 


\section{Acknowledgements}

Part of the work was done during the 2nd Workshop on Discrete Geometry and its Applications, held at Instituto de Matemáticas UNAM, Oaxaca, Mexico, September 2009. We thank all participants for useful discussions.

R.F-M and D. L. partially supported by Conacyt of Mexico, grant 153984. D. F-P. partially supported by grants 168277 (CONACYT, Mexico) and IA102513 (PAPIIT, UNAM, Mexico). J. U. partially supported by CONACyT of Mexico, grant CB-2012/178379.

\section{References}

G. Araujo, A. Dumitrescu, F. Hurtado, M. Noy, and J. Urrutia. On the chromatic number of some geometric type Kneser graphs. Computational Geometry: Theory and Aplications, 32(1):59-69, 2005.

I. Bárány and Z. Füredi. Empty simplices in Euclidean space. Canadian Mathematical Bulletin, 30:436-445, 1987.

I. Bárány and G. Károlyi. Problems and results around the Erdős-Szekeres convex polygon theorem. In Revised Papers from the Japanese Conference on Discrete and Computational Geometry, JCDCG '00, pages 91-105, London, UK, 2001. Springer-Verlag. ISBN 3-540-42306-0.

I. Bárány and P. Valtr. Planar point sets with a small number of empty convex polygons. Studia Scientiarum Mathematicarum Hungarica, 41:243-266, 2004.

C. Bautista-Santiago, J. Cano, R. Fabila-Monroy, D. Flores-Peñaloza, H. González-Aguilar, D. Lara, E. Sarmiento, and J. Urrutia. On the diameter of a Geometric Johnson type graph. In Proceedings of the 26th European Workshop on Computational Geometry, pages 61-64, 2010.

T. Denley. The odd girth of the generalised Kneser graph. European Journal of Combinatorics, 18(6):607-611, 1997.

A. Dumitrescu. Planar sets with few empty convex polygons. Studia Scientiarum Mathematicarum Hungarica, 36 : 93-109, 2000.

T. Etzion. On the nonexistence of perfect codes in the Johnson scheme. SIAM Journal on Discrete Mathematics, 9 (2):201-209, 1996.

R. Fabila-Monroy and C. Huemer. Covering islands in plane point sets. In Proc. XIV Encuentros de Geometría Computacional ECG2011, pages 213-216, Alcalá de Henares, Spain, 2011.

C. Godsil and G. Royle. Algebraic Graph Theory. Springer Verlag New York, 2001.

J. D. Horton. Sets with no empty convex 7-gons. Canadian Mathematical Bulletin, 26:482-484, 1983.

M. Katchalski and A. Meir. On empty triangles determined by points in the plane. Acta Mathematica Hungarica, 51: 323-328, 1988.

J. Matoušek. Lectures on Discrete Geometry. Springer Verlag New York, 2002.

F. Muga, J. Caro, H. Adorna, and G. Baes. On the wide-diameter of the Johnson graph $J(n, k)$. The Loyola Schools Review, 1:78-88, 2001

P. Valtr. On the minimum number of empty polygons in planar point sets. Studia Scientiarum Mathematicarum Hungarica, 30:155-163, 1995. 